Instituto Internacional de Investigación y Desarrollo Tecnológico Educativo INDTEC, C.A.

DOI: https://doi.org/10.29394/scientific.issn.2542-2987.2017.2.3.2.31-52

OAI-PMH: http://www.indteca.com/ojs/index.php/Revista Scientific/oai

\title{
Competencias de Escritura en Estudiantes de la Carrera de Educación (Caso: Universidad Pedagógica Experimental Libertador. Mérida, Venezuela)
}

Autoras: Gisela Consolación Quintero Chacón Universidad Nacional de Educación, UNAE

gishelinaq@hotmail.com

Chuquipata, Ecuador

Teresa de Jesús Molina Gutiérrez Universidad Nacional Abierta, UNA teresaj.molina@gmail.com Mérida, Venezuela Ana Rosa di Gravia de Grabadillo Universidad Nacional de Educación, UNAE adigravia@gmail.com Chuquipata, Ecuador

\section{Resumen}

En este trabajo se presentan los resultados parciales de una investigación llevada a cabo sobre las competencias de escritura de los estudiantes de la carrera de Educación de la Universidad Pedagógica Experimental Libertador en Venezuela. El objetivo general de la investigación consistió en identificar las características de tipo sintáctico, semántico y pragmático inherentes a la construcción de los textos elaborados por los estudiantes. El método empleado para la interpretación de los datos fue el Análisis del Discurso, el cual permitió abordar los procesos comunicativos para describir sus estructuras a nivel textual. Se adecuó la tabla de dificultades de Serrano y Mostacero que contiene ciento cincuenta y siete (157) dificultades y se llevó a veinticuatro (24); distribuidas de la siguiente manera: once (11) correspondientes a la sintaxis, ocho (8) a la semántica y cinco (5) a la Pragmática. Los resultados obtenidos demuestran que los rasgos en la dimensión semántica y pragmática fueron valorados de regular y mal. Estos resultados permitirán diseñar un programa integral que actuará a favor de propuestas innovadoras para el mejoramiento del proceso de escritura en la formación de profesionales de la carrera de Educación.

Palabras clave: escritura académica; semántica; pragmática. 
Writing Skills in Students of Education.

(Case: Universidad Pedagógica Experimental Libertador. Mérida, Venezuela)

\section{Abstract}

This paper presents the partial results of a research conducted on the writing skills of students of the Education at the Pedagogical University Experimental Libertador in Venezuela. The overall objective of the research was to identify the characteristics of syntactic, semantic and pragmatic types of texts produced by students. The method used for the interpretation of the data was discourse analysis, which allowed communication processes addressing their structures to describe them textually. The difficulty table was adapted from Serrano and Mostacero containing one hundred fifty seven (157) difficulties was reduced to twenty-four (24); distributed as follows: eleven (11) for syntax, eight (8) to semantics and five (5) to the Pragmatic. The results show a high percentage of features in the semantic and pragmatic dimension valued regular and bad. These results will help to design a comprehensive program that will act in favor of innovative proposals for improving the writing process in the training of professionals in Education career.

Keywords: writing academic; semantics; pragmatics. 


\section{Introducción}

Son múltiples las razones acerca de la relevancia de los procesos de lectura y escritura, pero especial atención adquiere su enseñanza. En ambos procesos se asientan los principios educativos, y en éstos a su vez, se cultivan valores, conductas. En resumen, se desarrollan capacidades y habilidades esenciales para el crecimiento personal, social y profesional.

Ser un escritor competente es una tarea fundamental en la vida académica y de acuerdo con Bolívar y Beke (2011) tanto la lectura como la escritura, son prácticas sociales y modos de interacción social con una repercusión determinante en los procesos de formación académica; no son procesos transferibles indiscriminadamente, sino que cumplen funciones muy específicas en las diversas áreas del saber.

Investigadores como Arnáez (2009), Del Valle (2007), Martínez (2002) han ahondado sobre la importancia de la enseñanza de la lectura y de la escritura, en la necesidad de renovar las propuestas pedagógicas, así como han concebido a la primera como la capacidad para comprender significados y a la segunda como una habilidad basada en procesos. En el mismo sentido, Hernández (2009) da relevancia a los estudios sistemáticos sobre los aportes de corrientes teóricas como la psicolingüística, el análisis del discurso y el constructivismo.

Afirma Carlino (2005): "la escritura alberga un potencial epistémico, es decir no resulta sólo un medio de registro o comunicación, sino un instrumento para desarrollar, revisar y transformar el propio saber" (pág. 411). Por esta razón la escritura ocupa un sitial preponderante en los procesos de formación académica y por este mismo motivo los responsables de orientar ese aprendizaje deben involucrarse, muy comprometidamente, en todo lo que implique mejorar las estrategias de enseñanza.

En este contexto, en opinión de Carlino (2005), es esencial conocer en las comunidades académicas cómo se intercambian prácticas y saberes que 
son inherentes a cada área o disciplina del conocimiento y cómo en cada una de estas prácticas se emplea un discurso particular, además de la necesidad de adquirir las competencias escritoras requeridas en dichas comunidades del conocimiento, es fundamental comprender el discurso de las diversas ramas del saber, pues cada campo disciplinar exige un acercamiento particular con los fines y propósitos de cada área, con el uso del lenguaje y con sus características.

Esta problemática nos sitúa en el concepto de alfabetización académica, al respecto Carlino (2003) "señala el conjunto de nociones y estrategias necesarias para participar en la cultura discursiva de las disciplinas, así como en las actividades de producción y análisis de textos requeridas para aprender en la universidad" (pág. 410). Para Radloff y de la Harpe (2000), este concepto alude al proceso que permite incorporarse a una comunidad científica.

Acerca de la problemática relacionada con las escasas habilidades de los estudiantes para desempeñarse como un escritor competente en los estudios superiores investigadores como Bolívar y Beke (2011), Beke y Bruno (2000), Serrano y Mostacero (2014), Carlino (2003, 2004), han hecho aportes sobre la comunicación académica y científica, partiendo desde estos supuestos existe un elevado número de estudiantes e investigadores que no siempre pueden darle a sus textos el estilo y las características textuales, discursivas y pragmáticas exigidas, en muchos casos, se asume erróneamente que estas competencias se adquieren en los niveles superiores del sistema educativo, contextos en los se ofrecen menos posibilidades para favorecer el contacto de los jóvenes con las diversidades textuales.

En correspondencia con la problemática planteada el objetivo del estudio fue: identificar las competencias de tipo sintáctico, semántico pragmático inherentes a la construcción de los textos elaborados por los estudiantes de Educación. 


\section{Teoría y conceptos}

Con relación a la fundamentación teórica que sustenta esta ponencia, es preciso señalar la importancia de "La escritura académica en Venezuela. Investigación, reflexión y propuestas" cuyos compiladores son Serrano y Mostacero (2014). Se alude a la urgente necesidad de encontrar herramientas pedagógicas para facilitar el desarrollo del lenguaje y pensamiento.

Por su parte, investigadores como Cassany (1999), han difundido importantes estudios sobre las dificultades que interfieren en los procesos de producción y comprensión de textos orales y escritos. En tanto, Bolívar y Beke (2011), Martínez (2002), se han interesado en las características del discurso en los entornos científicos, profesionales y académicos.

También es un referente ineludible es Carlino (2003, 2004, 2005), quien Ilama la atención sobre el concepto de alfabetización académica para significar las especificidades de los discursos atendiendo a las distintas disciplinas científicas, pues cada una de ellas dispone de "una particular cultura escrita, con prácticas e interpretación y producción textual características" (Carlino, 2003, pág. 413).

Finalmente, tenemos a Cordero (2000) y Gramajo (2002), quienes abordan las dificultades escriturales presentes en los textos argumentativos elaborados por escolares. Los datos reportados por estos investigadores ratifican que los estudiantes confrontan dificultades al momento de precisar su intención argumentativa, estructurar su argumentación y adecuarse a las necesidades del contexto.

Van Dijk (1978) señala que las categorías que conforman un texto y que permiten identificarlo, no son sólo categorías estructurales sintácticas, sino también conceptuales semánticas; a las que se le deben sumar las categorías pragmáticas relacionadas con las funciones específicas de los tipos de textos. Para describir los aspectos sintácticos, semánticos y pragmáticos del texto, es 
necesario tener en cuenta los siguientes niveles de análisis propuestos por Van Dijk: macroestructura, superestructura y microestructura.

Macroestructura: Es de naturaleza semántica y constituye una representación abstracta de la estructura global del significado, asimismo se apoya en categorías y reglas de tipo general y convencional que los hablantes conocen implícitamente.

Superestructura: Constituye un esquema al que el texto se adapta y permite reconocer su estructura global. Las superestructuras "determinan el orden (la coordinación) global de las partes del texto" (Van Dijk, 1978, p. 150). Las mismas facilitan analizar los conceptos sobre un tema determinado, y sus interrelaciones, así como los diferentes tipos de conectores o elementos gramaticales.

Microestructura: Conforma las estructuras locales del texto y ayuda a profundizar en el uso de oraciones subordinadas, causales, consecutivas, adversativas condicionales y sus respectivas conjugaciones, para explicitar las relaciones lógico-argumentativas.

En este contexto los retos que se plantean a los estudiantes, no sólo tienen que ver con las técnicas de composición del texto, sino con las exigencias académicas de cada área del conocimiento y con las expectativas del receptor.

\section{Metodología}

El diseño de la investigación es descriptiva-transversal bajo el paradigma cuantitativo para el análisis porcentual y cualitativo para el análisis del discurso, se buscó caracterizar el tipo de dificultades (rasgos) presentes en los textos escritos. Los resultados de este trabajo forman parte de un proyecto de investigación más amplio, se analiza, desde el punto de vista 
lingüístico, discursivo y textual, los textos producidos por estudiantes universitarios en cuatro países: Ecuador, Perú, Cuba y Venezuela. En este avance, se presentan los resultados derivados del análisis de las dificultades identificadas en 41 textos elaborados por 41 estudiantes de la carrera de Educación en las menciones de: Inicial, Integral y Especial de la Universidad Pedagógica Experimental Libertador en Venezuela.

En cuanto a la recolección del corpus se aplicó una prueba diagnóstica a 41 estudiantes, la misma se organizó en tres partes. 1: datos generales. 2: lectura de texto para responder preguntas de comprensión lectora, 3: elaborar un texto argumentativo (tres párrafos) a partir de un tópico dado y 3.1: redactar un texto (tres párrafos) considerando la imagen presentada.

Para la descripción de los textos, se empleó una escala de 5 niveles de evaluación (Excelente, Muy bien, Bien, Regular y Mal), para cada rasgo. Esta escala permitió valorar tres dimensiones textuales: dimensión sintáctica (integrada por 11 rasgos), dimensión semántica (contentiva de 6 rasgos) y la dimensión pragmática (estructurada en 7 rasgos). Al respecto es necesario precisar que sólo por razones metodológicas se abordan por separado cada una de las dimensiones referidas.

Técnica de análisis de datos: El método empleado para la interpretación de los datos fue el Análisis del Discurso (Van Dijk, 1999), el cual permite abordar los procesos comunicativos para describir sus estructuras a nivel textual, la unidad de análisis es el texto. El análisis se apoyó en perspectivas cualitativas y cuantitativas de la investigación; en lo que atañe a la primera visión se analizaron las cualidades lingüísticas del texto mediante el Análisis de Contenido. La segunda postura se utilizó la estadística descriptiva para ponderar la presencia o ausencia de los rasgos establecidos como criterios de valoración Además del análisis cualitativo los datos se analizaron mediante la estadística descriptiva, se empleó el cálculo de la 
media y el análisis porcentual para destacar los aspectos más importantes relativos a las dificultades con mayores índices porcentuales en los textos estudiados.

\section{Resultados}

\subsection{Análisis de la Dimensión Semántica}

Para iniciar el aparte correspondiente a la evaluación de los rasgos de la Dimensión Semántica, se analizó el nivel macroestructural del texto, el cual de acuerdo con Van Dijk (1978) contiene las estructuras textuales de tipo global de naturaleza semántica, éstas integran la representación abstracta de la naturaleza global de significado del texto. Mientras que las secuencias cumplen las condiciones de coherencia lineal, los textos no sólo han de cumplir estas condiciones, sino además las de coherencia global.

- Coherencia semántica entre las ideas principales de las oraciones del párrafo (coherencia global).

En primer término, se discute sobre los resultados obtenidos para el indicador Coherencia semántica entre las ideas principales de las oraciones del párrafo (coherencia global). El 58,5\% de los participantes obtuvo la calificación de Regular, entonces, hay limitaciones para lograr la propiedad lógico semántica del discurso, a partir de estos resultados se puede calificar de regular el desarrollo proposicional lógico, la proposiciones también muestran dificultades para relacionarse semántica y lógicamente.

- Coherencia semántica entre las ideas centrales de los párrafos (coherencia local).

En cuanto al indicador Coherencia semántica entre las ideas centrales de los párrafos (coherencia local), es preciso establecer que el 
53,6\% de los participantes se ubicó en la escala Regular, en consecuencia, existe un manejo no satisfactorio de las destrezas para generar coherencia a las estructuras locales, las cuales además de integrar mecanismos como deixis textual, contienen, de modo especial, las proformas lexicales y éstas actúan como un tipo de guía para inferir los significados presentes en un texto.

En la siguiente muestra se observa la transgresión de las reglas en cuanto a la coherencia local, hay dificultad para relacionar intratextualmente las oraciones mediante los recursos como sustitución, elipsis, proformas gramaticales, no hay jerarquización de ideas principales ni secundarias y el párrafo se torna ambiguo e incomprensible debido a la transgresión de la regla de la no contradicción (Lomas, 1996), ésta evita la aparición de elementos contradictorios; en consecuencia, es difícil reconstruir la macroestructura.

"El aborto es un proceso donde por distintas razones suele suceder en algunos casos pasa porque la mujer no se encuentra preparada fisicamente [físicamente] y tiende a complicarsele [complicársele] el embarazo teniendo una placenta muy debil [débil] y es en este caso donde algunas veces se le es practicado un legrado. En otros casos el aborto es provocado por la mujer ingiriendo pastillas que hacen provocarlo matando asi [así] a un ser que viene, quitando el derecho de vida donde hoy por hoy es un delito.

- Existencia de marcas o elementos de enlace semántico entre las distintas secciones o apartados del texto.

Con respecto al indicador Existencia de marcas o elementos de enlace semántico entre las distintas secciones o apartados del texto, el $41,4 \%$ de la muestra estudiada se ubicó en la escala Mal, en virtud de lo cual, los informantes no disponen de estrategias adecuadas para establecer el enlace semántico entre los párrafos que integran el texto, es decir, se quiebra la propiedad semántica que hace posible la interpretación de cada oración a 
partir de las otras oraciones que conforman el texto. Las marcas que permiten lograr el enlace entre las proposiciones, secciones o apartados del texto son los conectores, de ello se deriva su importancia en el establecimiento de instrucciones que desentrañan el sentido de los enunciados.

\section{- Logra el cierre semántico}

En cuanto al rasgo Logra el cierre semántico, los resultados reportaron los mayores índices de aparición $(51,2 \%)$, para la alternativa Mal, ello indica que la muestra carece de las estrategias lingüísticas que permiten lograr que el texto sea una unidad lingüística, lo que necesariamente reclama la presencia del "cierre semántico y comunicativo" (Bernárdez. 1987, pág. 85).

En la muestra siguiente se observa una serie de transgresiones a las reglas textuales: inadecuación ortográfica, no hay orden (jerarquía, cronología, otros) en el desarrollo de las proposiciones, ausencia de conectores, por lo tanto, es muy difícil entender la organización del tópico del discurso, no hay modo de saber si existen relaciones de causa-consecuencia, de concesión, de oposición, de explicación, entre otras.

Todo ello incide en que la progresión temática, (avance de la información), no se efectúe acertadamente, tampoco hay coherencia entre los párrafos y menos aún una unidad temática; en consecuencia, no se alcanza el cierre semántico o conclusión. Al estar ausente el valor final que se tendría que establecer a partir de la idea inicial, es decir el cierre semántico, se impide que se consolide la argumentación.

Como lo indica Bernárdez (1987), una de las características más relevantes del texto es su cierre semántico y comunicativo, así como su coherencia; la suma de éstas revela la intención comunicativa de generar un texto con sentido completo. De estas cualidades carecen la mayoría de los textos analizados se puede comprobar en el siguiente ejemplo. 
"La imagen da a entender que dios todo lo puede es el salvador y redentor de todo mal

Si todos creemos y tenemos amor y fuerza sobre nuestro dios todo será mejor facil [fácil] de resolver siendo el problema que sea solo [sólo] tener confianza y fé [fe]. Asumiendo cada fraude y derrota".

En los textos analizados el material textual presentado no logra concretar el significado pretendido por el escritor y tampoco tiene sentido para el lector en función de la intencionalidad, ni de los factores situacionales.

\subsection{Análisis de la Dimensión Pragmática}

La dimensión pragmática alude al requerimiento del texto de encuadrar en una situación comunicativa e involucra componentes extralingüísticos: los participantes, situacionalidad (uso personal, público, académico, profesional). Como lo señala Blum-Kulka (2001) el éxito en la comunicación no sólo depende del conocimiento de la gramática de las palabras de la lengua, "en su interpretación intervienen una multiplicidad de factores, entre los cuales se encuentran la familiaridad con el contexto, las marcas de entonación y los supuestos culturales" (pág. 67).

- Evidencia de la intención comunicativa del autor.

En lo que respecta al indicador Evidencia de la intención comunicativa del autor, el 53,6\% de la muestra se ubicó en la calificación de Regular, de modo que no hay habilidades apropiadas que evidencien la intencionalidad de quien escribe el texto, la misma se hace presente cuando a través de la actitud el autor logra su objetivo comunicativo. 


\begin{abstract}
Al considerar el siguiente ejemplo, en el cual se solicitó a los estudiantes elaborar un texto argumentativo, se analiza si se logra a intención comunicativa del autor:

“El aborto: En la medicina se entiende por aborto toda expulsión del feto natural o provocada en el periodo no viable de su vida intrauterina, es decir cuando no tiene ninguna posibilidad de sobrevivir. Si esa expulsión del feto se realiza en periodo [período] no viable pero antes del termino [término] del embarazo se denomina parto prematuro tanto si el feto sobrevive como si muere.
\end{abstract}

Entre los que aparecen marcadores para cohesionar, están ausentes la mayoría de los signos de puntuación, se acerca a definiciones aisladas sobre el aborto. Al ser un texto argumentativo, la intención del autor sería hacer valer un punto de vista, por lo tanto, debería exponer razones, enfrentar, matizar afirmaciones para lograr convencer sobre su tesis. Como se observa están ausentes las marcas lingüísticas para construir un lenguaje que además de desarrollar un tópico, se presume es conocido por los estudiantes, puedan manejar estrategias para persuadir sobre la aceptabilidad de la tesis a demostrar,

\title{
- Uso de las palabras del español estándar
}

En cuanto al Uso de las palabras del español estándar, el 46,3\% de los participantes se ubican en la calificación de Regular, hay un escaso manejo de términos técnicos, así como se transgreden las convenciones de la lengua escrita.

El siguiente ejemplo constituye una muestra de la transgresión de las normas más elementales del lenguaje elaborado (lengua estándar): 
"El aborto es una persona que de verdad no se podria [podría]_guscar [juzgar] de alguna manera porque hay veces que lo hacen por saber que su hijo viene a llevar muchas necesidades y otra porque deberia [debería] ser que se tiene que cuidar y proteguer [proteger] por cuidados de precausion [precaución]".

- Uso del lenguaje directo.

Por otra parte, el indicador Uso del lenguaje directo (denotación), reportó el $48,7 \%$ de empleo para la escala Mal, no existe ningún manejo del recurso lingüístico para emplear significados nítidos y de fácil comprensión para los interlocutores. En la mayoría de los textos estudiados se reporta un elevado empleo de la primera persona del plural, (en el ejemplo anterior aparecen verbos en primera persona y posesivos), lo cual constituye una manera de marcar lingüísticamente la subjetividad del autor.

\section{Discusión}

Resulta evidente ante la exigencia que plantea la escritura de reelaborar el conocimiento, pero en la muestra analizada no se escogen las mejores estrategias retóricas para organizar y presentar los contenidos: no se logró establecer relaciones oportunas, ni adecuada articulación, por ello, la significación es ambigüa. En general, los estudiantes no cuentan con competencias para hacer un uso estratégico de la capacidad cognitiva, la intención comunicativa, los objetivos perseguidos, el tipo de texto, el dominio del tema y de las características de la audiencia.

Es relevante precisar que al ubicarse en la dimensión pragmática se alude a los aspectos textuales que hacen posible evidenciar la intención que persigue el autor del texto al dirigirse a los lectores (Bernárdez, 1987). De modo que esas habilidades están bastante disminuidas y se confrontan 
importantes barreras para construir discursos ajustados a los requerimientos pragmáticos de cada práctica social. Es escaso el conocimiento que facilita identificar la práctica social en la que se ubican los textos, ni tampoco las reglas que los estructuran. Asimismo, hay poca consciencia de que escribir involucra valores para ambos interlocutores porque es una actividad que se inserta en un entorno sociocultural (Flower y Hayes, 1987).

Para concluir también es importante precisar que estos resultados coinciden con los de Atienza y López (1994) cuando señalan que, al analizar los aspectos contextuales del discurso académico, existen dificultades para escoger la información y organizarla, hay falta de adecuación de los tópicos y no se tiene en cuenta al interlocutor. En el mismo sentido, corroboran los hallazgos de Flower y Hayes (1987) al precisar la dificultad de los universitarios para desarrollar sus escritos teniendo en cuenta la perspectiva del lector.

\section{Conclusiones}

- Aunque el estudiante al escribir requiere "recordar la ortografía de miles de palabras, tiene que distinguir las sutiles normas de puntuación, es necesario que conozca la estructura, los registros y las formas de la cohesión de los textos que quiere escribir" (Cassany, 1999, pág. 65). Las dificultades encontradas en la muestra indican un uso muy precario de competencias lingüísticas básicas, consolidadas ya en el nivel universitario. Esto evidenció una evaluación de regular y mal en el manejo de las dimensiones semántica y pragmática.

- Con respecto a las dificultades referidas a la dimensión semántica (cuatro (4) rasgos se evaluaron como Regulares y dos (2) rasgos se consideraron Mal (para este artículo se presentaron sólo 3 rasgos) los de mayor relevancia. Esas insuficiencias se relacionan con la generación de textos en los que las ideas secundarias no logran aportar 
la información necesaria para elaborar la idea principal, por tanto, no hay una macroestructura que funcione como la idea común a través del desarrollo del texto.

- La fragmentación originada por la falta de conectores interfiere con la continuidad del sentido o linealidad semántica, en consecuencia, el lector no puede obtener el significado global, ni se le aportan los recursos para guiar el proceso inferencial, no hay posibilidad de hacer homogénea la superficie del texto porque está cargada de ambigüedad.

- En lo referente a la dimensión pragmática cuatro (4) rasgos se consideraron Regulares y los tres (3) restantes se calificaron Mal, (para este artículo se presentaron sólo 3 rasgos). Se evidencia que no se cuenta con la capacidad estratégico-pragmática para elaborar los textos identificando la práctica social del contexto, ni se aplican adecuadamente las reglas.

- Los datos reportados en este estudio se encuentran correspondencia con los resultados obtenidos por otros investigadores quienes también abordaron las competencias escritoras de los estudiantes universitarios. Ilich y Morales (2004), determinaron la escasa competencia discursiva de los estudiantes para producir textos expositivos y argumentativos. En cuanto a Fuenmayor, Villasmil y Rincón (2008) concluyeron que los textos expositivos producidos por los estudiantes son poco informativos e incoherentes, por su parte, Iglesia, García y Mainegra (2006), sostienen que lograr la progresión temática y la coherencia presenta dificultades en la mayoría de los textos elaborados en distintos niveles del sistema educativo y Molina y López (2015), plantean la incapacidad de los estudiantes de maestría para elaborar el planteamiento del problema de la tesis de grado, ello 
debido al escaso conocimiento del género discursivo propio de cada una de las disciplinas abordadas en los estudios de postgrado.

- En consecuencia, las carencias evidenciadas en el corpus analizado, limita a estos estudiantes las posibilidades ciertas de valerse eficientemente del potencial epistémico de la escritura, el cual de acuerdo con Carlino (2003) "no resulta sólo un medio de registro o comunicación sino también deviene en un instrumento para desarrollar, revisar y transformar el propio saber" (pág. 411) y menos aún pueden relacionar el saber sobre la temática abordada con el conocimiento de los requerimientos situacionales de la redacción: fines y destinatario.

- Finalmente, los resultados obtenidos nada satisfactorios, es necesario tener muy en cuenta la complejidad que revisten los procesos de lectura y escritura, pues como lo indica Carlino $(2004,2005)$ comprensión, producción de textos y conocimiento son inseparables, asimismo no hay que perder de vista al tipo de estudiantes pues se enfrentan a una cultura ajena, tanto en las prácticas lingüísticas, como en el conocimiento de las áreas disciplinares pedagógicas que integran el currículo universitario. En consecuencia, se hace urgente plantear propuestas integrales que contribuyan realmente a mejorar la escritura académica de los estudiantes que cursan la carrera de Educación no sólo del caso estudiado sino extensivo a otras instituciones de Venezuela como de Latinoamérica.

\section{Referencias}

Arnáez, P. (2009). La lectura y la escritura en la educación básica. Educere, vol. 13. $n^{\circ} 45,289-298$. Recuperado de:

http://www.scielo.org.ve/scielo.php?script=sci arttext\&pid=S1316$\underline{49102009000200004}$ 
Atienza, E. y López, C. (1994). La progresión temática en el discurso académico. Ponencia presentada ante el XII Congreso Nacional de la Asociación Española de Lingüística Aplicada AESLA. Nuevos Horizontes de la Lingüística Aplicada, 101. Barcelona: Universidad Autónoma de Barcelona. Recuperado de:

https://www.upf.edu/pdi/dtf/carmen lopez ferrero/aesla.pdf

Bernárdez, E. (1987). Lingüística del texto. Madrid: Arco/Libros.

Beke, R. y Bruno, E. (2000). "El uso de estrategias para la elaboración de resúmenes de textos en el ámbito académico". En: Boletín de Lingüística No. 15, pp. 19-35.

Blum-Kulka, S. (2001). Pragmática del discurso. En Van Dijk, T. (Comp.). EI discurso como interacción social. Estudios sobre el discurso II. Una introducción multidisciplinaria, pp. 20-67. Barcelona: Gedisa.

Bolívar, A. y Beke. R. (Comp.) (2011). Lectura y escritura para la investigación. Caracas: Universidad Central de Venezuela.

Carlino, P. (2003). Alfabetización académica: Un cambio necesario, algunas alternativas posibles. EDUCERE, Investigación, 6(20), 409420.

Carlino, P. (2004). El proceso de escritura académica. EDUCERE, Artículos Arbitrados, 8(26), 321-327.

Carlino, P. (2005). Escribir, leer y aprender en la universidad. Una introducción a la alfabetización académica. Buenos Aires: Fondo de Cultura Económica.

Cassany, D. (1999). Construir la escritura. Barcelona: Paidós.

Cordero, M. (2000). El componente tesis en los textos argumentativos escolares. Signos, 33(48), 87-96.

Del Valle, L. (2007). Enseñanza de la lengua escrita en la primera etapa de Escuela Básica. Estudio de caso descriptivo sobre educadores 
venezolanos. Barcelona: Tesis doctoral no publicada de la Universidad de Barcelona.

Flower, L. y Hayes, J. (1987). The cognition of discovery: Defining a rhetorical problem. College composition and Comunication, 31, 21-32.

Fuenmayor, G., Villasmil, Y., Rincón, M. (2008). Construcción de la microestructura y macroestructura semántica en textos expositivos producidos por estudiantes universitarios de LUZ. Letras 50(77), 189-219. Recuperado de:

http://www.scielo.org.ve/scielo.php?script=sci arttext\&pid=S0459$\underline{12832008000200007}$

Gramajo, A. (2002). Modelos mentales en la comprensión del texto argumentativo. Memoria del Congreso Internacional. La Argumentación: lingüística, lógica, retórica, pedagogía, pp. 740-747. Buenos Aires: Universidad de Buenos Aires.

Hernández, G. (2009). Escritura académica y formación de maestros. ¿Por qué no acaban las tesis? Tiempo de Educar, Vol. 10, Núm. 19, pp. 1140 México: Recuperado de:

http://www.redalyc.org/articulo.oa?id=31113164002

Iglesia, T., García, M. y Mainegra, D. (2006). El tratamiento de a la progresión temática y a la coherencia en la construcción de textos. Mendive, 4(15), 20-40.

Ilich, E., y Morales, O. (2004). Análisis de textos expositivos producidos por estudiantes universitarios desde la perspectiva lingüística discursiva. Educere, 8, 333-345. Venezuela.

Lomas, C. (1996). La educación lingüística y literaria en la enseñanza secundaria. Barcelona: Horsori.

Martínez, S. M. (2002). Estrategias de lectura y escritura de textos. Perspectivas teóricas y talleres. Universidad del Valle. Colombia. 
Molina, T. y López, A. (2015). El planteamiento del problema: un problema de competencias de escritura o de desconocimiento de la cultura discursiva de las disciplinas. Ponencia presentada en el I Jornada de Escritura y Lectura como Prácticas Académicas y Sociales. Universidad Pedagógica Experimental Libertador. Mérida.

Radloff, A., y De la Harpe, B. (2000). Helping student develop their writing skills a resource for lecturers. En Flexible Learning for a Flexible Society, Actas del ASET, Higher Education Research and Developmente in South Asia. Conference. Toowoomba, Queensland.

Serrano, S., y Mostacero, R. (2014). La escrita académica en Venezuela: Investigación, reflexión y propuestas. Acción Pedagógica, (26), 187193. Mérida, Venezuela: Universidad de Los Andes.

Van Dijk, T. (1978). La ciencia del texto. Barcelona: Paidós.

Van Dijk, T. (1999). El Análisis crítico del discurso. In Anthropos, 186, pp. 


\section{Gisela Consolación Quintero Chacón \\ e-mail: gishelinaq@hotmail.com}

Venezolana. Doctora en Ciencias Pedagógicas.

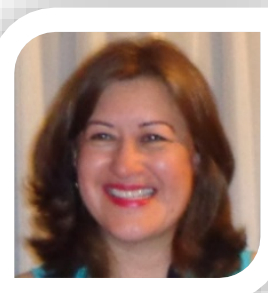

Máster en Gestión Universitaria. Especialista en

Psiconeurolingüísti. Licenciada en Letras. Jubilada de

la Universidad Nacional Experimental de Guayana.

Docente de pregrado y postgrado. Ha participado en eventos nacionales como ponente y como

conferencista. En los actuales momentos es asisitente de la Coordinación de Posgrado de la Universidad Nacional de Educación (UNAE) en Ecuador y dirige el proyecto de investigación: Caracterización de la producción escrita de los estudiantes de Educación y de las concepciones para la enseñanza de la escritura de cuatro países: Ecuador, Venezuala, Cuba y Perú. 


\section{Teresa de Jesús Molina Gutiérrez}

e-mail: teresaj.molina@gmail.com

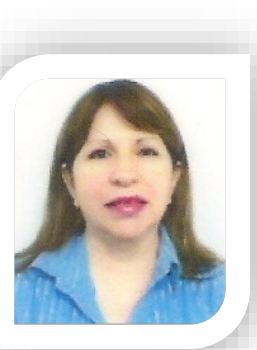

Magister en Lingüística, en Literatura y Doctora en

Lingüística, profesora de pregrado y postgrado, con categoría de Titular en la Universidad Nacional Abierta, coordinadora del Grupo de Investigación: Innovación en Educación y Medios Instruccionales de la UNA. Ponente, en eventos de investigación nacional e internacional, investigadora PEI. Línea de investigación: Análisis del Discurso, Enseñanza de la Lengua. 


\section{Ana Rosa di Gravia de Grabadillo}

e-mail: adigravia@gmail.com

Profesora Asociada, jubilada de la Universidad

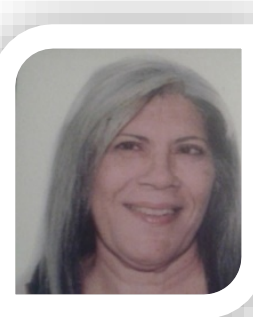

Nacional Experimental de Guayana (UNEG), actualmente Profesora-Investigadora, Agregado I, en la Universidad Nacional de Educación (UNAE). Lic. en Psicología en la Universidad Central de Venezuela (UCV), Magister en Investigación Educativa en la Universidad de Carabobo (UC) y Doctora en Ciencias de la Educación en la Universidad Nacional Experimental Simón Rodríguez (UNESR). Miembro activo de Linea-I, investigadora en el proyecto "Éxitus" y en el Proyecto "Estrategias innovadoras para la Educación Inclusiva", adscrito a la Coordinación de Investigación de la UNAE.

El contenido de este manuscrito se difunde bajo una Licencia de Creative Commons ReconocimientoNoComercial-Compartirlgual 4.0 Internacional 